\title{
CAN BIG DATA MACHINES ANALYZE STOCK MARKET SENTIMENT?
}

Vasant Dhar

Editor-in-Chief

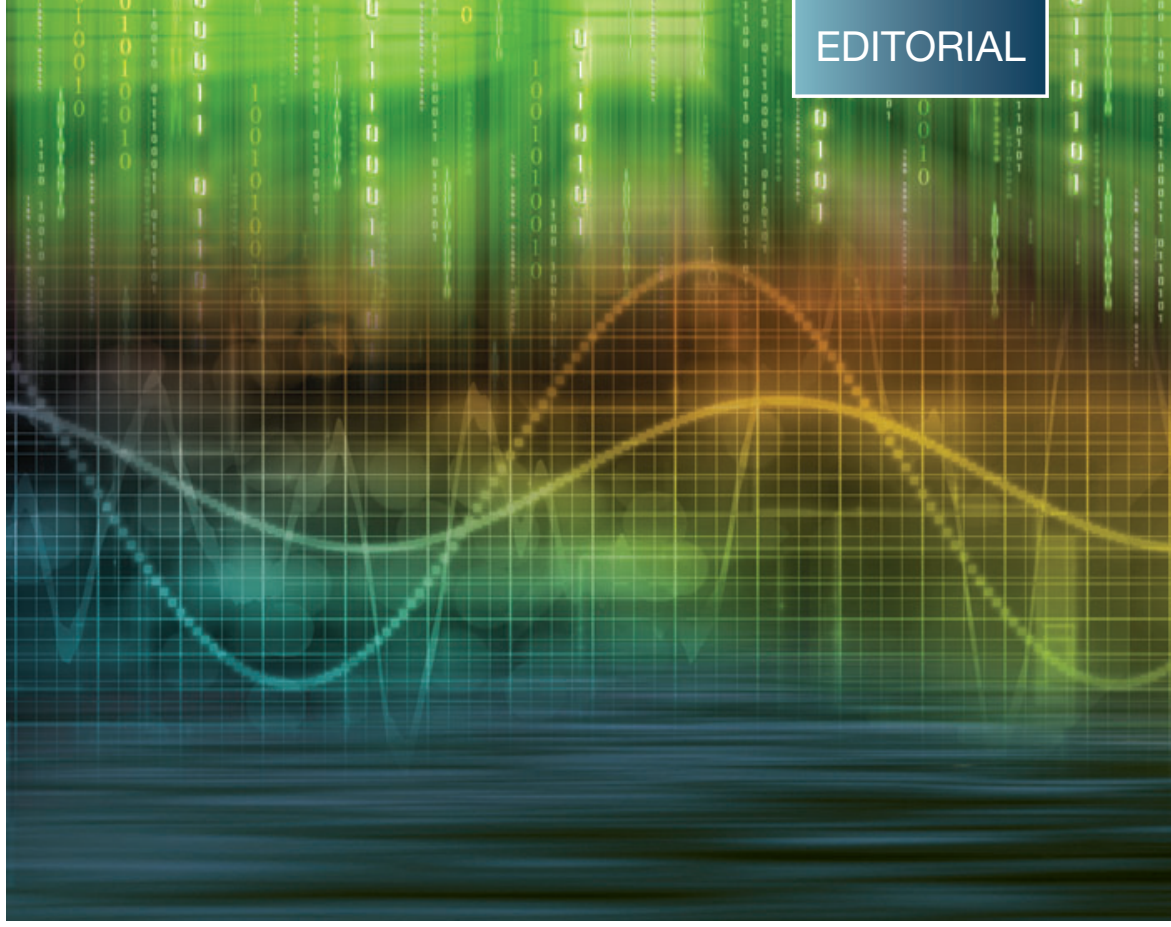

\section{Abstract}

Do the massive amounts of social and professionally curated data on the Internet contain useful sentiment about the market that "big data machines" can extract systematically? If so, what are the important challenges in creating economic value from these diffuse sources? In this commentary, I delve into these questions and frame the challenges involved using recent market developments as an illustrative backdrop.

It IS INTRIGUING to CONSIDER how the vast amounts of professionally curated and socially generated unstructured "big data" might be harnessed to yield useful insights into financial markets. Proponents of "wisdom of the crowds" contend that such data may contain within it some key insights about specific variables of interest. Can we find any evidence of this wisdom in such data?

Most existing macroeconomic indicators of the economy are derived from government data and surveys, and established ratings agencies. Data about companies mostly come from their financial statements, earnings announcements, and various types of periodic disclosures by management and agencies that rate or comment about them. Investment professionals rely on these data sources to assess the strength of the economy and to make investment recommendations or decisions. In contrast, the growing volumes of unstructured data on the Internet contain a new type of intellectual and emotional content that is less well understood at the moment. Is there any evidence that such data might contain useful information for investors or policy makers?
To address this question, let's start by considering Figure 1, which plots the S\&P500 market index from January 2006 through October 31,2014, as the blue time series, versus the frequency of the phrase "market correction" as the red series reported from Google Trends over the same period. ${ }^{1}$

The numbers on the graph (right scale) reflect how many searches have been done for a particular term, relative to the

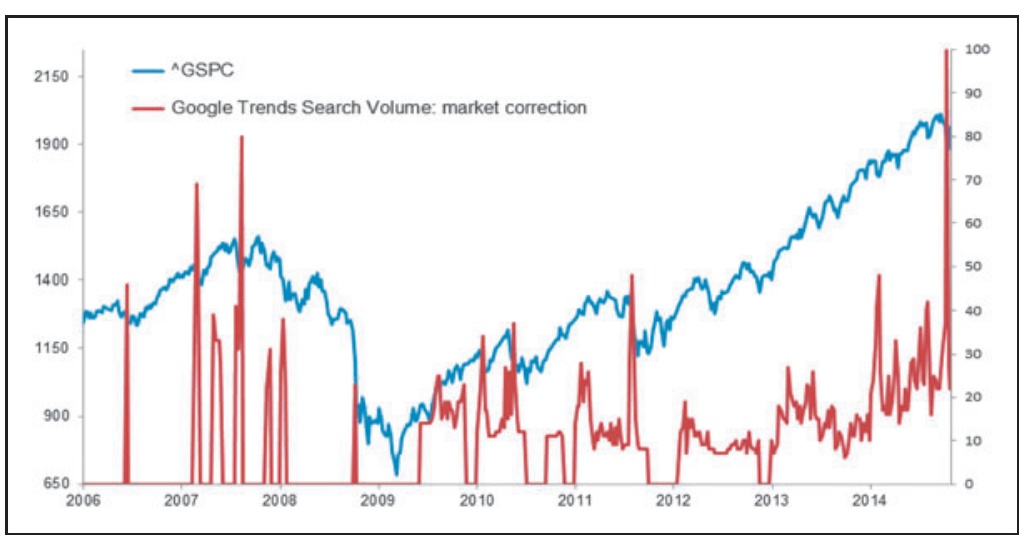

FIG. 1. S\&P 500 and search volume for "market correction" on google trends. 
total number of searches done on Google over time. They don't represent absolute search volume numbers, because the data is normalized and presented on a scale from 0 to 100 . Each point on the graph is divided by the highest point and multiplied by 100 . Google shows 0 when enough data is not shown.

It is readily apparent that the recent use of the phrase "market correction" is at an all-time high. The current peak is significantly higher than those that preceded the market collapse of 2008 when the S\&P500 dropped by roughly $60 \%$ from its peak in October 2007 to its trough in March 2009. Could we interpret the pattern as indicating a heightened sense of nervousness about the market?

In a New York Times economic commentary on October 18, 2014, Robert Shiller asked whether the abrupt decline of $6 \%$ that week signaled the beginning of a bear market. Shiller used the metaphor of a "thought virus" to describe the market, arguing that the stock market is driven by popular narratives, some of which can spread by contagion. Shiller's thought virus is similar in many ways to a meme, which is defined as "an idea, behavior, or style that spreads from person to person within a culture." 2 Some of these are pernicious, like the "debt ceiling crisis." This latter virus took hold between May 2 and June 15, 2011, when the S\&P dropped by over $7 \%$ for fear that Congress would not raise the debt ceiling in time and the U.S. government would default on its debt. The virus "died" when Congress raised the ceiling on July 31, 2011. Interestingly, however, the market had one of its worst days on August 4, which was after the Congress action, driven by a different virus that had been quiescent: fears of a global economic slowdown expressed in this August 4 headline in CNN Money: "The Dow tumbled 512 points — its ninth deepest point drop ever — as fear about the global economy spooked investors."3

Sound familiar to what we are hearing today?! The market continued to grind down until October 3, 2011, after which it rallied as the "global slowdown" meme abated. Arguably, this type of meme doesn't ever die out completely but remains dormant, rising up periodically when conditions are hospitable. These memes include fears of recession, inflation, interest rates (hikes or cuts), political risks, and, these days, fear of terrorist acts. The challenge is whether these memes can be identified and measured accurately enough over time to provide useful ex ante sentiment indicators that presage socioeconomic activity.

Consider zooming in on the last 90 days of Figure 1 and also integrating some additional news-based information from this period. The chart in Figure 2 (now focusing on Monday, August 4, 2014, to October 31,2014) shows the S\&P500 along with the daily average positive and negative sentiments computed from an al- gorithm that used 13,269 business news stories on Reuters that appeared over that period. The sentiment measures were calculated using the General Inquirer (GI) methodology* in which every word is classified as belonging to one or more predefined categories such as positive, negative, strong, and weak. A simple proportion is calculated for each category based on a count of words in the category relative to the total words in the sample. The GI methodology has been shown to be useful in some studies as a simple first-order approach for sentiment extraction. ${ }^{4}$ In our calculation, we normalize the individual sentiment percentages to yield a type of normalized $Z$-score ${ }^{\dagger}$ of positive and negative sentiment for each day. We use the numbers for illustration purposes only, recognizing the several possible deficiences in our crude calculation of sentiment.

The chart is broken into three periods separated by the vertical black bars:

(1) August 4 and September 18, 2014, where a steadily rising market flattens out. During this time, the S\&P500 rose by roughly $5 \%$ and the positive and negative sentiment scores both oscillated around 1 .

(2) A sharp drop of roughly 6\% in the S\&P500 between September 18 and October 15, 2014, in response to fears of a global slowdown. The positive sentiment dropped by roughly $30 \%$, while the level of negative sentiment drifted slightly higher with three pronounced peaks of increasing magnitude at weekly intervals-on September 27, October 4, and October 11 (sentiments tend to peak on Saturdays, which is not altogether surprising as the media take stock, no pun intended, of the week).

(3) A sharp reversal of over $8 \%$ for the S\&P500 between October 15 and October 31 as the market made new highs. Interestingly, the average negative sentiment stayed above 1 during the rebound while the positive sentiment has remained lower than its pre-"global slowdown” level.

How might we interpret this type of data, such as a drop in positive sentiment? One interpretation could be that while there are always doomsayers, when the countervailing optimists drop out of the discussion, it bodes ill for the market. A contrary interpretation, however, could be that the low-positivesentiment scenario coupled with a healthy level of negative sentiment is indicative of a key segment of the investment community having already taken money off the table. In this latter narrative, this "sidelined" money can be put back into the market to provide and support a market rise in the near term. (This could, for example, explain the market rise in the third period above despite the low positive sentiment.) A slightly different variant of this view could hypothesize that low negative sentiment coupled with high positive sentiment would represent

*General Inquirer's Harvard-IV-4 classification dictionary is used to measure positive and negative sentiment.

${ }^{\top}$ This is equal to the mean of the scores divided by their standard deviation. 


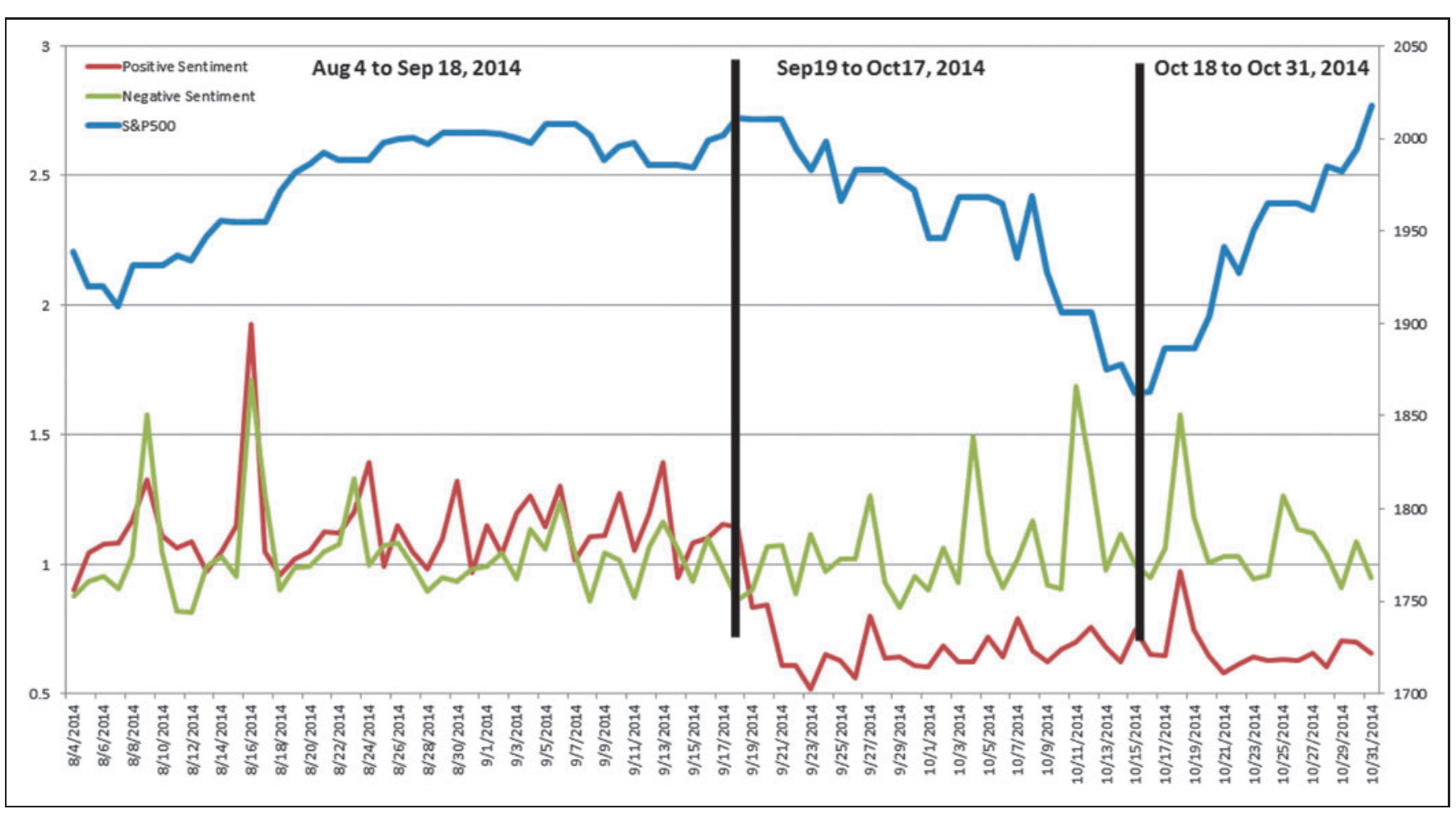

FIG. 2. S\&P 500 vs. positive and negative sentiment in business news stories.

a heightened state of risk in which investors are fully invested as a herd, but where bad news can spark a rapid correction.

The possibility of multiple theories suggests that we must dig deeper and more methodically into the data to try to address such questions and identify characteristic memes, and to measure which ones are getting stronger or weaker. This is indeed what big data enables computers to do, namely, to posit reasons for patterns that are not readily apparent to humans who don't always have the wisdom to ask exactly the right question.

There are several major challenges in fleshing out such a meme contagion theory. For starters, can these memes be codified, identified, and tracked ex ante to provide normative guidance, rather than just retrospective commentary? Can this be done automatically with some reliability? If so, can the detection of such memes be done in a manner as to add economically value beyond what is done today? Answering these questions requires characterizing changes in sentiment: if the professional media is essentially reflecting what's already happened in the market, it is less useful than if it describes the

drivers of what is happening, which could be predictive of futures states and not just descriptive of the past.

Interestingly, we have the computational machinery, although far from perfect, for automatic extraction of "hot topics" from unstructured data ${ }^{5,6}$ that could be used to identify memes as they evolve over time. If this technology is good enough today, or will be soon, the major remaining challenges revolve around data quality, problem formulation, interpretation, and integration.

\section{"THERE ARE SEVERAL MAJOR CHALLENGES IN FLESHING OUT SUCH A MEME CONTAGION THEORY."}

Fundamentally, the current streams of news and business data are of variable quality and potentially biased by the incentives of the authors or data providers. While the professional news media might at least attempt to provide objective reports, information from the officers and PR departments of corporations often has a strong optimistic bias. ${ }^{*}$ Data about companies and markets from investment professionals can also be heavily biased. For example, equity analysts' ratings of public companies appear to be dominated by herd effects ${ }^{7}$ where those with lesser status or from less reputable investment houses might

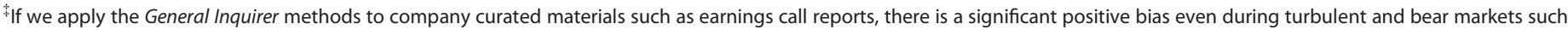
as the one between 2007 and 2009. 
gravitate toward consensus forecasts. ${ }^{8}$ (Individual investors also tend to exhibit herd behavior as noted by several studies in finance where momentum has been a longstanding "anomaly."9)

Even more serious concerns exist about the reliability of the vast amounts of data emanating from social media such as Twitter, Facebook, and LinkedIn, which, arguably, represent crowds to a larger extent than the professional news media. Research to date on the quality of such data has observed significant herding biases in some social media outlets including online ratings. ${ }^{10}$ Ratings of music, ${ }^{11}$ books, ${ }^{12}$ and other products appear to show evidence of multiple sources of influence and exhibit anchoring and herding biases and, in general, tend to be favorably biased. ${ }^{13}$ Testimonials can also be explicitly manipulated, as is evident in the hotel industry, ${ }^{14}$ where there are strong economic incentives for favorable reviews. Misinformation can also spread rapidly on the Internet, as occurred on April 23, 2013, when hackers took over the Associated Press Twitter account and tweeted that two explosions at the White House had injured the president. ${ }^{15}$ The tweet was retweeted more than 3,000 times within a few minutes before Twitter took the account offline, while the S\&P500 plunged almost a percent and then recovered when the hoax was discovered.

The above cautions notwithstanding, one might still argue that as social media mature we should be able to correct for the credibility and inherent biases in their associated data. In other words, we should be able to grade the credibility of data from the various sources-governmental, news, business, and social media-perhaps in that order, and use it accordingly. The challenge then is to automatically associate these data correctly with the higher level themes such as "secular stagnation," "inflation," and so on. If we are able to identify such high-level themes as they emerge, and can measure and relate them to the financial markets, we may be able to build credible explanatory and predictive systems that are far better at systematically estimating various types of future risks and returns-both large and small—than are the current generation. Such systems may also be far more effective at identifying major systemic risks and market corrections. However, this raises the tantalizing but unsettling question of whether these systems themselves will become susceptible to- or become the subject of - a new species of "algorithmic viruses" or memes.

As of this writing, for example, concerns about Europe have resurfaced to include the narratives of stress test failures of certain European banks and/or the need for aggressive quantitative easing. Could this environment of depressed positive sentiment be a fertile one in which a pessimistic meme can take hold (particularly if a contingent of the investment community were to become nervous and take risk off the table)? Or will it be overshadowed by the generally optimistic market trend to date?

Regardless of how the future pans out, professional investors cannot ignore the emergence of a new and critical class of "factor" in their investment models, namely, big data-based sentiment, and the growing capability for market participants to extract and measure it reliably. This new type of factor, which is very different from indices such as the Michigan consumer sentiment index, may well join the ranks of key determinants of investment performance, alongside traditional factors such as value and momentum that have been used for decades on Wall Street.

For data scientists, the challenge is how to create the machinery that deals with the challenges associated with handling the torrent of increasing unstructured data emanating from the various sources. We are at the very early stages of making sense of this type of big data. As tempting as it might be, we should not be too quick to accept that any one of these sources has predictive power. Rather, it is worth digging deeper and integrating multiple sources of unstructured with traditional data so that we are able to explain complex social, political, and economic phenomena and perhaps find ex ante indicators of risks, returns, and other variables of interest. The challenge has just begun.

\section{References}

1. About trends graphs. Available online at https://support .google.com/trends/answer/4355164?hl = en\&rd = 1

2. Meme. Available online at http://en.wikipedia.org/wiki/ Meme

3. Censky A. 2011. Stocks: Worst day since 2008 financial crisis. Available online at http://money.cnn.com/2011/08/ 04/markets/markets_newyork/

4. Tetlock P, Saar-Tsechansky M, Macskassy S. More than words: Quantifying language to measure firms' fundamentals. J Finance 2008; LXIII.

5. Macskassy S, Hirsh H, Provost F, et al. Intelligent Information Triage. The 24th Annual International Conference on Research and Development in Information Retrieval (SIGIR), September 2001.

6. Blei D, Lafferty J. 2003. Topic models. Available online at www.cs.princeton.edu/ blei/papers/BleiLafferty2009.pdf

7. Moses H. Accuracy, usefulness and the evaluation of analysts' forecasts. Int J Forecast 2003; 19:417-434.

8. Graham J. Herding among investment newsletters: Theory and evidence. J Finance 1999; LIV. 
9. Jegadeesh N, Titman S. Returns to buying winners and selling losers: Implications for stock market efficiency. J Finance 1993; 48:65-91.

10. Aral S. The problem with online ratings. MIT Sloan Management Review, Winter 2014.

11. Frank RH. 2012. Luck vs. skill: Seeking the secret of your success. Available online at www.nytimes.com/2012/08/05/ business/of-luck-and-success-economic-view.html?_r $=0$

12. Chevalier J, Mayzlin D. The effect of word of mouth on sales: Online book reviews. J Market Res 2006; 43:345-354.

13. Hu N, Zhang J, Pavlou PA. Overcoming the J-shaped distribution of product reviews. Commun ACM 2009; 52:144-147.
14. Mayzlin D, Dover Y, Chevalier J. Promotional reviews: An empirical investigation of online review manipulation. The American Economic Review, September 2013.

15. Kelly H. 2013. The power of one wrong tweet. Available online at www.cnn.com/2013/04/23/tech/social-media/ tweet-ripple-effect/

Address correspondence to:

Vasant Dhar

Editor-in-Chief

Big Data 